\title{
Forum
}

\section{«Wenn ich gross bin, will ich Lacan verstehen» Ein kleiner Erfahrungsbericht zum Projekt «Klinisches Arbeiten mit Lacan in und zwischen zwei Sprachen»}

Tamara Lewin (Zürich)

Im Herbst 2014 haben sich einige Analytikerinnen und Analytiker aus Deutschland, Frankreich und der Schweiz unter der Leitung von Rony Weissberg, Annemarie Hamdad, André Michels und Patrick Landman zusammengetan, um gemeinsam ein klinisches Wochenendseminar durchzuführen und ein weiterführendes Projekt, ein «work in progress», zu lancieren mit dem Ziel, sich künftig gemeinsam auf dem Hintergrund der klinischen Theorie Lacans über Fälle auszutauschen. Seither treffen drei mal pro Jahr - jeweils abwechselnd in Zürich, Paris und Berlin - Analytiker_innen, die in der Kultur der lacanschen Analyse gelernt haben und arbeiten, auf solche, die die «freudsche Sprache» sprechen und im deutschsprachigen Kulturraum arbeiten, um in kontinuierlicher Übersetzung in beiden Sprachen über ihre Praxis zu sprechen. Die Arbeitstagungen sind jeweils in drei Blöcke gegliedert, in denen auf ein theoretisches Referat eine Falldarstellung und -besprechung folgt.

Aus den Beiträgen und deren schriftlichen Übersetzungen wird jeweils ein Arbeitsheft zusammengestellt, das die Grundlage für das nächste Treffen bildet und auch einen Neu- oder Wiedereinstieg jederzeit möglich macht. Bisher lag der thematische Schwerpunkt auf der Deutung bzw. l'interprétation. Für das Treffen im Juni 2016 ist der Beginn eines neuen Zyklus vorgesehen, der sich dem Thema «Übertragung und Trieb» widmet.

Am Anfang meiner Auseinandersetzung mit Lacan stand eine Mischung aus trotziger Ablehnung und einer vagen Ahnung, in seinem Denken etwas zu finden, was mir in anderen Theorien fehlte. Die ehrfurchteinflössende Aura des grossen Meisters und Guru, die Mythen und die gängigen Floskeln um seine Lehre bzw. 
deren Unzugänglichkeit hatten mich immer wieder unangenehm berührt und eher Abwehr ausgelöst.

Im Rahmen meiner Weiterbildung hielt ich mich so zunächst einmal von Lacan fern, was am PSZ nicht weiter schwierig ist. Bis Rony Weissberg zusammen mit Barbara Langraf einen explizit klinisch ausgerichteten Kurs zu Lacan anbot, den ich während zwei Semestern besuchte.

Als ich von der trinationalen Initiative erfuhr, hatte ich also schon das eine oder andere über Lacan gehört und befand mich, ob es mir gefiel oder nicht, in genau jener vielzitierten Bewegung zwischen Faszination (= diffuses Gefühl, dass genau das gemeint ist, was man immer schon dachte, aber nie hätte formulieren können) und Frustration (= Gefühl unüberbrückbarer Distanz der Theorie zum eigentlich Erfahrbaren). Die Aussicht auf eine andere Form des Zugangs zu diesen beiden Seiten der wohl selben Medaille erschien mir vor diesem Hintergrund unmittelbar attraktiv, und so meldete ich mich zur ersten Arbeitstagung an und sagte auch gleich zu, am Tag zwei eine von drei Falldarstellungen zu übernehmen. «Einfach so wie gewohnt» könne diese aussehen, antwortete Rony auf meine Frage nach allfälligen Vorgaben oder Erwartungen dazu.

So fand ich mich also an jenem Freitagabend mit einigen Weiterbildungskolleginnen und -kollegen zum ersten Teil der Tagung ein und war gespannt zu sehen, was wohl für Leute aus Paris und Berlin angereist kämen. Besonders interessiert war ich auch daran, einen persönlichen, atmosphärischen Eindruck der Weiterbildungsinstitutionen und -teilnehmenden unserer Nachbarländer zu gewinnen - eine Neugier, die unbefriedigt bleiben musste, da zu meinem Erstaunen kaum junge Menschen zur Tagung kamen.

Nach der Begrüssung und dem ersten Referat wurden Stühle gerückt und gemäss der Planung der Gastgeber_Innen ein innerer Kreis mit ca. 8 Stühlen gebildet. Die Erläuterungen zu Tradition und Zweck dieses «Zürcher Modells» konnten der Irritation und Verwunderung, die von den fragenden Gesichtern der ausländischen Gäste (besonders derer aus dem Westen) abzulesen waren, erst einmal keinen Abbruch tun. Es folgte die erste Falldarstellung, bestritten durch eine erfahrene Analytikerin aus Paris, und jetzt wurde mir ihr Staunen schon verständlicher: Der Fall war spannend erzählt, die berichteten Interventionen brilliant, die Theoretisierung des Geschehens schlüssig - eine publikationsreife Präsentation, die des Schutzes eines «inneren Kreises» nicht bedurfte. Mir wurde flau, sollte ich doch ein paar Stunden später selbst von meiner Arbeit berichten. Aber es gab kein Zurück mehr, und so versuchte ich am nächsten Tag, möglichst in der mir gewohnten Weise - das heisst, möglichst theoriefrei und geleitet vom erlebten 
Beziehungsgeschehen - von den Sitzungen mit einem Patienten zu berichten, den ich auf einer psychiatrischen Akutstation während einiger weniger Wochen begleitete und der mir einiges an Kopfzerbrechen bereitete.

Nachdem ich am Ende dieses Tages meinem unmittelbaren Fluchtimpuls eine Denkpause entgegensetzte, war ich bald der Überzeugung, dass es sich lohnen würde, an der Sache dran zu bleiben. Zu Recht, denn tatsächlich entwickelte sich im weiteren Verlauf zunehmend eine Vertrautheit, die es den Teilnehmenden möglich machte, sich gerade auch mit ihren Fragezeichen zu zeigen.

Die wohl wichtigste Erkenntnis an diesem ersten Treffen war für mich, dass ich mein Verständnis psychoanalytischen Arbeitens und vor allem des Nachdenkens und Sprechens darüber keineswegs als Allgemeingut verstehen kann. So etwa schien die Selbstverständlichkeit, mit der szenisches Verstehen oder das offene Nachdenken über meine eigenen Gegenübertragungsgefühle zu einer Fallbesprechung für mich dazugehören, andernorts befremdlich zu wirken. Und wiederum lerne ich von den Lacanianer_innen eine besondere Art und Weise, in der analytischen Situation auf die Sprache des Gegenübers zu hören, eine Art, die Wortwörtlichkeit ernst zu nehmen und mit ihr zu arbeiten.

«Work in progress» meint also deutlich mehr als die kontinuierliche Weiterarbeit an bestimmten thematischen Schwerpunkten. Der Progress bedeutet auch ein Sich-Einlassen auf eine interkulturelle Verständigung und Annäherung und eine höchst spannende Erarbeitung eines gemeinsamen Diskurses. Die Arbeitsatmosphäre hat sich so über die verschiedenen Treffen hinweg bereits deutlich verändert und bereichert und tut dies weiter, wozu nicht zuletzt wohl auch das Reisen der Gruppe und das Kennenlernen der jeweiligen institutionellen Heimat der Teilnehmenden beiträgt.

Die Treffen gestalten sich so als anregendes Forum, das eine intensive Auseinandersetzung und Begegnung mit unterschiedlichen Formen des klinischen Arbeitens in und mit Lacans Konzepten ermöglicht. Besonders wertvoll erlebe ich die Treffen aber nicht nur wegen des konsequent plastischen klinischen Bezugs durch die Arbeit mit Fällen, und das richtiggehende Eintauchen verdankt sich mehr als der verfügbaren Zeit. Die Zweisprachigkeit, das heisst die fortlaufende Übersetzung des Gesprochenen bei allen Beiträgen, die man sich auf den ersten Blick als störende Verzögerung und Hindernis des Denkens vorstellen könnte, erweist sich als grosse Bereicherung: Während den Übersetzungssequenzen entsteht mehr Raum für eigene Assoziationen und Fragen zum eben Gehörten. Vor allem aber werden einerseits immer wieder Aspekte des Materials durch den Prozess des Transfers in eine andere Sprache - mit all seinen Tücken - sichtbar, und 
andererseits entsteht durch das raschere Hervortreten unterschiedlicher Facetten ein besonderer Eindruck von Dreidimensionalität.

Der Austausch verspricht also, spannend zu bleiben. Zu hoffen wäre umso mehr, dass sich inskünftig noch etwas mehr junge Teilnehmehnde dazugesellen und so neben dem Austausch zwischen Kulturen dem intergenerationellen Aspekt noch etwas mehr Raum verschafft wird.

Die nächsten Termine:

17./18. Juni 2016: Zürich

23./24. September 2016: Paris 